\title{
Influencia del tipo de fundente en las propiedades de la porcelana de huesos
}

\author{
C. GARCÍA PORTILLO \\ Dpto. de Ciencia de Materiales. Escuela Cerámica. Manises. Valencia.
}

\begin{abstract}
En el presente trabajo se estudia la acción del calor sobre los constituyentes de la porcelana de huesos. Se formulan tres composiciones, en las que variamos el tipo de fundente.

Se situan dichas composiciones en el diagrama de fases $\mathrm{Ca}_{3}\left(\mathrm{PO}_{4}\right)_{2}-\mathrm{Al}_{2} \mathrm{O}_{3}-\mathrm{SiO}_{2}$; a la luz del cual se intenta deducir las fases cristalinas o vítreas presentes. Se estudia la influencia del fundente en las propiedades del producto obtenido.

Palabras clave: porcelana de huesos, fundentes, diagrama de equilibrio, propiedades físicas.

\section{Influence of the type of mekting on the properties of bone porcelain}

In our present work we are studying the effect of heat on the constituents of bone porcelain. We have formulated three compositions, in which we varied the type of melting. We placed these compositions in the phases diagram $\mathrm{Ca}_{3}\left(\mathrm{PO}_{4}\right)_{2}-\mathrm{Al}_{2} \mathrm{O}_{3}-$ $\mathrm{SiO}_{2}$; with which we intend deduce the presents crystalline or vitrious phases. We are studying the influence of the type of melting on the properties of the obtained product.
\end{abstract}

Key words: bone china, fluxes, phase equilibrium, physical properties.

\section{INTRODUCCIÓN}

La porcelana de huesos se fabricó por primera vez en Inglaterra, a mediados del siglo dieciocho. Se atribuye a Josiah Spode en 1789 (1) el perfeccionamiento de la pasta. En 1794 se comenzó su fabricación a escala industrial, en Stoke-on-Trent, (Inglaterra). En este país la porcelana de huesos fue copiada por todos los fabricantes de porcelana. En la actualidad, se produce porcelana de huesos a escala industrial en Gran Bretaña, Suecia y Japón.

La principal aplicación de la porcelana de huesos es la fabricación de vajilla de mesa. Esto se debe a sus buenas propiedades físicas y estéticas. La porcelana de huesos es muy resistente, blanca, resonante y traslúcida.

La formulación clásica de la pasta de porcelana de huesos es: $50 \%$ de ceniza de huesos, $25 \%$ de caolín (china clay) y $25 \%$ de piedra de Cornwall (1).

El vidriado que se aplica a las piezas de porcelana de huesos se basa en borosilicato de plomo, que se cuece en segunda cocción a temperatura inferior a la del soporte. Este se cuece a $1250{ }^{\circ} \mathrm{C}$ y el vidriado lo hace aproximadamente a $1150{ }^{\circ} \mathrm{C}(1)$.

\section{REACCIONES QUE OCURREN DURANTE LA COCCIÓN}

St. Pierre (2), dice que a temperaturas inferiores a $1000{ }^{\circ} \mathrm{C}$ las reacciones que tienen lugar en la porcelana de huesos son las mismas que las que ocurren en otras pastas triaxiales. Por encima de $1000{ }^{\circ} \mathrm{C}$ las reacciones que sufre la porcelana de huesos son específicas de este material.
El efecto del calor sobre la ceniza de huesos se puede expresar como sigue:

$$
\mathrm{Ca}(\mathrm{OH})_{2} \cdot 3 \mathrm{Ca}_{3}\left(\mathrm{PO}_{4}\right)_{2} \rightarrow \mathrm{CaO}+\mathrm{H}_{2} \mathrm{O}+3 \beta-\mathrm{Ca}_{3}\left(\mathrm{PO}_{4}\right)_{2}
$$

Según St. Pierre (2), la ceniza de huesos comienza a descomponerse a $1000^{\circ} \mathrm{C}$. La experiencia de este trabajo nos indica que entre $1100{ }^{\circ} \mathrm{C}$ y $1200{ }^{\circ} \mathrm{C}$ se observa una elevada pérdida de peso. Esta pérdida de peso, a esta elevada temperatura, puede contribuir al elevado valor de la porosidad abierta a temperaturas tan sólo $50^{\circ} \mathrm{C}$ por debajo de la temperatura de maduración. El CaO liberado de la descomposición de la ceniza de huesos reacciona rápidamente con la caolinita deshidratada, para formar anortita, según la reacción siguiente:

$$
\mathrm{CaO}+\mathrm{Al}_{2} \mathrm{O}_{3} \cdot 2 \mathrm{SiO}_{2} \rightarrow \mathrm{CaO} \cdot \mathrm{Al}_{2} \mathrm{O}_{3} \cdot 2 \mathrm{SiO}_{2}
$$

Según Edwards (3), una tercera parte de la ceniza de huesos actúa como fundente, quedando el resto sin fundir y actuando como soporte de la pasta.. Según Wats (4), la ceniza de huesos sólo actúa como fundente a los conos 8 al 10 (1250 - $\left.1350{ }^{\circ} \mathrm{C}\right)$, pero no a temperaturas más bajas. La experiencia indica que la ceniza de huesos en cantidades elevadas como se utiliza en la porcelana de huesos, actúa fundamentalmente como material refractario, en cambio adicionada, en pequeñas cantidades a otras pastas de porcelana actúa como fundente enérgico (5).

Dinsdale (6) afirma que durante el calentamiento de la porcelana de huesos, parece que se dan los tres procesos siguientes: 
- Algo del CaO de los huesos se combina con el caolín para dar anortita: $\mathrm{CaO} \cdot \mathrm{Al}_{2} \mathrm{O}_{3} \cdot 2 \mathrm{SiO}_{2}$

- Todo el $\mathrm{CaO}$ restante da $\beta$ - fosfato tricálcico.

- Algo de $\mathrm{P}_{2} \mathrm{O}_{5}$ se combina con los otros materiales formando un vidrio.

El CaO que con más facilidad puede dar anortita es el procedente de la descomposición del $\mathrm{Ca}(\mathrm{OH})_{2}$, pero habrá que suponer que también se forma a partir de la descomposición del fosfato, ya que si no, la cantidad $\mathrm{CaO}$ sería insuficiente.

El anión fosfato que queda libre de la descomposición anterior se combina con parte de los óxidos presentes en las materias primas y forman un vidrio, cuya composición se indica en la tabla II.

De la alúmina que proporciona el caolín, una parte pasará a formar parte de la anortita, y la otra pasará a la fase vítrea.

Dinsdale (6) da en 1967 la relación de fases cristalinas y la composición de la fase vítrea, que puede verse en la Tabla I y II.

El término porcelana de huesos, se escribirá de forma abreviada como P.H.

\section{TABLA I.}

FASES PRESENTES EN LA PORCELANA DE HUESOS COCIDA

\begin{tabular}{|l|c|}
\hline Fases & $\%$ \\
\hline$\beta$ - fosfato tricálcico & 45 \\
\hline Anortita & 25 \\
\hline Vidrio & 30 \\
\hline
\end{tabular}

TABLA II.

PORCENTAJE DE ÓXIDOS EN LA FASE VÍTREA

\begin{tabular}{|l|r|}
\hline Óxidos & $\%$ \\
\hline $\mathrm{SiO}_{2}$ & 65 \\
\hline $\mathrm{Al}_{2} \mathrm{O}_{3}$ & 15 \\
\hline $\mathrm{P}_{2} \mathrm{O}_{5}$ & 5 \\
\hline $\mathrm{K}_{2} \mathrm{O}$ & 15 \\
\hline
\end{tabular}

En la matriz vítrea pueden esperarse además de los óxidos mencionados en la tabla II, pequeñas cantidades de $\mathrm{Na}_{2} \mathrm{O}$, y $\mathrm{MgO}$.

St. Pierre (7), centró su trabajo en el estudio de los diagramas de fases. En la fig. 1, se indica mediante un área trapezoidal, la región del diagrama donde se encuentran las composiciones de la porcelana de huesos. El autor indica que la microestructura de la porcelana de huesos esta constituida por cristales de $\beta$ - fosfato tricálcico y anortita insertos en la matriz vítrea.

Klause (8) indica la dificultad del método petrográfico, debido al tamaño muy pequeño de los cristales presentes.

Las fases cristalinas que podrían encontrarse en la porcelana de huesos, suponiendo que no hubiera fase vítrea serían: $\beta$-fosfato tricálcico, anortita, mullita y sílice. Esto lo muestra St. Pierre (9) en la fig. 2, al estudiar la compatibilidad tetraédrica entre el fosfato tricálcico - anortita - mullita - sílice. La mullita sería la fase menos abundante en una porcelana de huesos completamente cristalizada. La mullita sería a su vez la primera fase en disolverse al aumentar la temperatura.

Si la composición estudiada se encuentra en el campo primario de la sílice, ésta permanecerá sin disolver a la tempera-

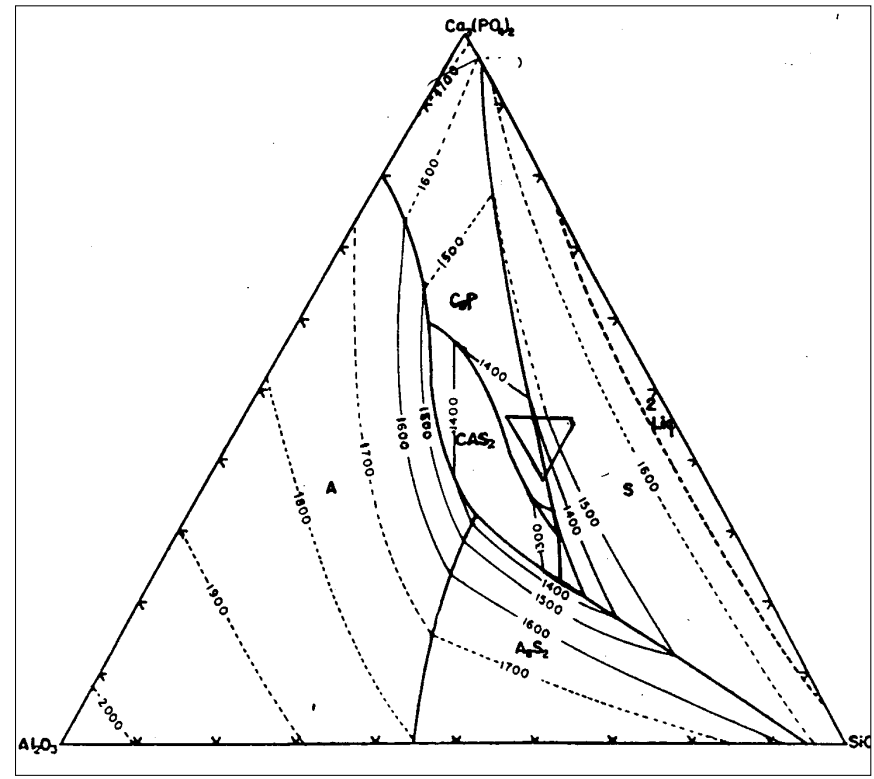

Fig. 1.- Diagrama de fases del sistema: $\mathrm{Ca}_{3}\left(\mathrm{PO}_{4}\right)_{2}-\mathrm{Al}_{2} \mathrm{O}_{3}-\mathrm{SiO}_{2}$.

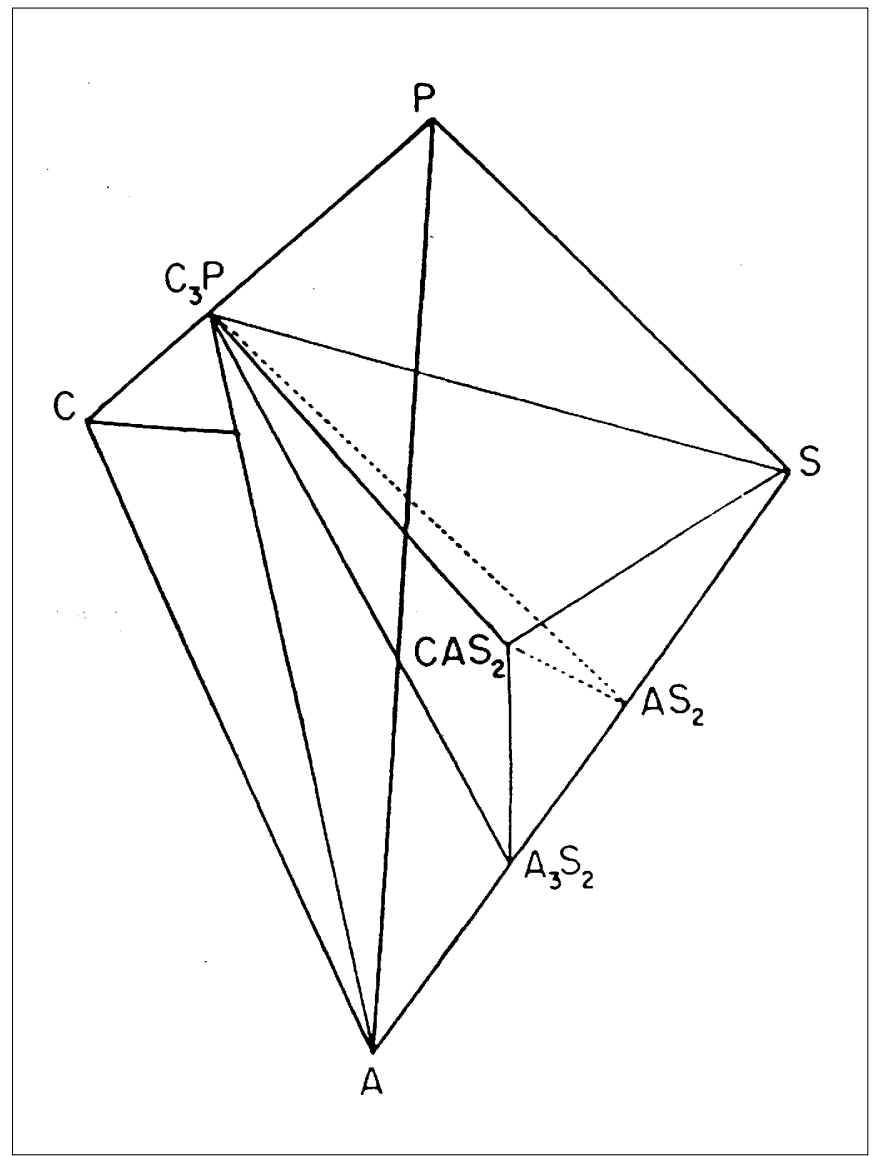

Fig. 2.- Diagrama cuaternario de equilibrio de fases: $\mathrm{CaO}-\mathrm{Al}_{2} \mathrm{O}_{3}$ $\mathrm{P}_{2} \mathrm{O}_{5}-\mathrm{SiO}_{2}$. 


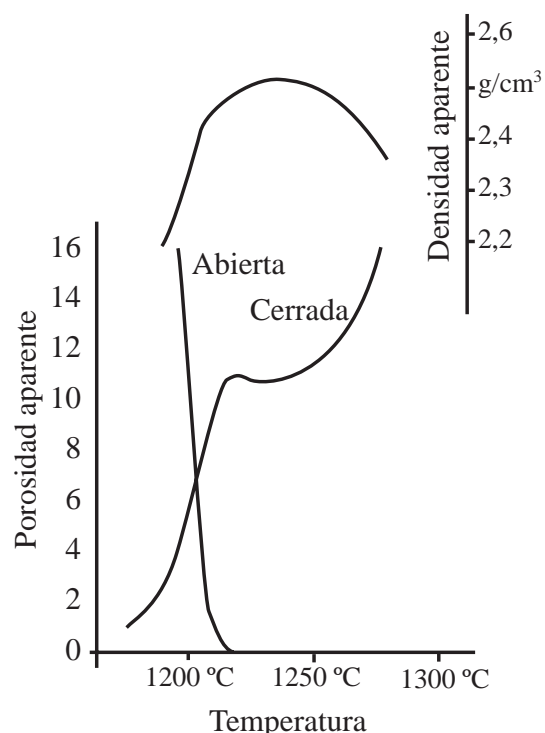

Fig. 3.- Diagrama de vitrificación de una Porcelana de Huesos.

tura de maduración de la pasta. Si a su vez el tamaño de partícula de la sílice es grueso, la disolución se verá doblemente impedida. Estas cantidades de sílice sin disolver afectarán a la textura y a la translucidez de la pasta.

La composición de la porcelana de huesos se encuentra cerca de un eutéctico. St. Pierre (7), a partir de sus estudios del diagrama de fases, indica que dicho eutéctico se encuentra entre el fosfato tricálcico, la anortita y la sílice, y que las proporciones de dichas fases, en el punto eutéctico, son respectivamente 10, 50 y $40 \%$. En cuanto se alcanza la temperatura eutéctica se forman cantidades sustanciales de vidrio, alrededor del 40 $\%$. La naturaleza extremadamente viscosa del líquido formado disminuye la elevada tendencia de la pasta a la deformación. La única forma de reducir la cantidad de líquido formado a la temperatura eutéctica es mediante el aumento de uno de los componentes, de modo que la composición de la pasta se aleje de la composición eutéctica. La mejor forma de preservar las características de la porcelana de huesos es mediante el aumento de la cantidad de ceniza de huesos, a expensas del mineral arcilloso, pero esto dificulta la manufactura de esta pasta, ya de por sí difícil de trabajar, debido a su baja plasticidad.

En otro estudio posterior St. Pierre (9), indica que las fases presentes en la composición eutéctica están en la siguiente proporción: $11 \%$ de fosfato tricálcico, $51 \%$ de anortita y $38 \%$ de sílice y que funde a $1290{ }^{\circ} \mathrm{C}$.

En las pastas cercanas a un eutéctico las reacciones siempre se dan muy rapidamente. Lo contrario ocurre en el sistema: sílice - alúmina - álcalis, presente en todas las otras pastas de cerámica blanca tradicional, en donde las reacciones son lentas y se extienden sobre un amplio rango de temperaturas.

Taylor (10) encontró, que al igual que en otros tipos de cerámica blanca, la sinterización de la porcelana de huesos, consiste en un proceso en dos etapas:

-Sinterización en estado sólido: Esta etapa comienza entre 700 ${ }^{\circ} \mathrm{C} 800{ }^{\circ} \mathrm{C}$, y se caracteriza por una marcada reducción de la superficie específica, y un cambio despreciable en la porosidad.

-Sinterización con fase vítrea: Esta etapa comienza de 1000 ${ }^{\circ} \mathrm{C}$ a $1150{ }^{\circ} \mathrm{C}$. Está caracterizada por la eliminación de los poros abiertos y va acompañada de una marcada contracción y de un aumento de la densidad aparente.

Al contrario de lo que ocurre en otras pastas vítreas, en la porcelana de huesos estas dos etapas están separadas suficientemente, lo que permite su identificación más fácilmente, que en el resto de pastas triaxiales tradicionales.

En la figura 3 puede verse el diagrama de vitrificación de una porcelana de huesos standard.

St. Pierre (2) analiza por rayos $X$, las fases presentes en una porcelana de huesos, calentada a diversas temperaturas, durante 20 minutos. En la tabla III, se expone de forma resumida este proceso.

TABLA III

CONSTITUYENTES DE LA PORCELANA DE HUESOS.

\begin{tabular}{|l|l|}
\hline Temp. $\left({ }^{\circ} \mathrm{C}\right)$ & \multicolumn{1}{|c|}{ Constituyentes } \\
\hline Sin cocer & Hidroxiapatito (H), Caolín $(\mathrm{C})$, Feldespatos $(\mathrm{F})$, Cuarzo $(\mathrm{Q})$, Mica $(\mathrm{M})$ \\
\hline 600 & El caolín se ha descompuesto. \\
\hline 800 & El pico de la mica se hace mucho más débil. El pico del feldespato disminuye. \\
\hline 1000 & El hidroxiapatito comienza su descomposición a $\beta$-fosfato tricálcico. \\
\hline & $\begin{array}{l}\text { El CaO, liberado del hidroxilapatito reacciona con el caolín deshidratado, para } \\
\text { dar anortita. }\end{array}$ \\
\hline 1200 & $\begin{array}{l}\text { El hidroxilapatito esta totalmente descompuesto. } \\
\text { El cuarzo está notablemente disuelto. }\end{array}$ \\
\hline 1300 & El cuarzo continúa disminuyendo. Posiblemente la anortita esta disolviéndose. \\
\hline 1370 & Sólo existe $\beta$-fosfato tricálcico. Los demás constituyentes se han disuelto. \\
\hline
\end{tabular}

\section{DESARROLLO EXPERIMENTAL}

Las materias primas utilizadas han sido: ceniza de huesos, caolín standard, nefelina sienita de Noruega, feldespato potásico de Sudáfrica.

La ceniza de huesos, caolín standard, nefelina sienita de Noruega, feldespato potásico de Sudáfrica han sido suministrada por Vicar. La pegmatita ha sido provista por la firma Sanchís Penella.

Se han preparado 3 pastas de porcelana de huesos, en las que se ha variado el tipo de fundente.

En la tabla IV se indican los análisis químicos de las materias primas utilizadas y en la tabla $\mathrm{V}$ se indican las composiciones de las pastas estudiadas.

TABLA IV

ANÁLISIS QUÍMICO DE LAS MATERIAS PRIMAS.

\begin{tabular}{|l|l|l|l|l|l|l|}
\hline A. Q. & $\begin{array}{l}\text { Caolín } \\
\text { S. } \mathbf{P .}\end{array}$ & $\begin{array}{l}\text { Ar. } \\
\text { H. } \mathbf{M .}\end{array}$ & $\begin{array}{c}\text { Ceniza } \\
\text { H. }\end{array}$ & $\begin{array}{c}\text { Peg- } \\
\text { matita }\end{array}$ & $\begin{array}{c}\text { Nef. } \\
\text { Sien.N }\end{array}$ & $\begin{array}{c}\text { Fel. K. } \\
\text { Sudaf. }\end{array}$ \\
\hline $\mathrm{SiO}_{2}$ & 47,81 & 49,25 & 0,34 & 75,21 & 57,00 & 67,10 \\
\hline $\mathrm{Al}_{2} \mathrm{O}_{3}$ & 36,85 & 31,16 & 0,05 & 14,47 & 23,80 & 18,26 \\
\hline $\mathrm{Fe}_{2} \mathrm{O}_{3}$ & 0,70 & 1,21 & 0,05 & 0,12 & 0,10 & 0,06 \\
\hline $\mathrm{TiO}_{2}$ & 0,00 & 0,91 & 0,01 & 0,05 & 0,00 & 0,01 \\
\hline $\mathrm{CaO}$ & 0,10 & 0,20 & 54,00 & 0,32 & 1,10 & 0,12 \\
\hline $\mathrm{MgO}$ & 0,30 & 0,30 & 0,97 & 0,22 & 0,00 & 0,04 \\
\hline $\mathrm{K}_{2} \mathrm{O}$ & 1,69 & 2,11 & 0,02 & 5,70 & 9,10 & 11,59 \\
\hline $\mathrm{Na}_{2} \mathrm{O}$ & 0,10 & 0,30 & 1,38 & 3,58 & 7,80 & 2,45 \\
\hline $\mathrm{P.P.C}$. & 12,45 & 14,57 & 1,19 & 0,33 & 1,10 & 0,37 \\
\hline $\mathrm{P}_{2} \mathrm{O}_{5}$ & 0,00 & 0,00 & 41,98 & 0,00 & 0,00 & 0,00 \\
\hline
\end{tabular}


TABLA V

\section{COMPOSICIÓN DE LAS PASTAS.}

\begin{tabular}{|l|l|l|l|}
\hline M P & P 1 & P 2 & P 3 \\
\hline Caolín S. P. & 15 & 15 & 15 \\
\hline Ar. H. M. & 10 & 10 & 10 \\
\hline Ceniza H. & 50 & 50 & 50 \\
\hline Pegmatita & 25 & 0 & 0 \\
\hline Nef. Sien. N. & 0 & 25 & 0 \\
\hline Fel. K. Sudaf. & 0 & 0 & 25 \\
\hline
\end{tabular}

Las materias primas han sido tamizadas todas, por tamiz de 0,5 mm de luz de malla. Después de la dosificación adecuada, se han molido por vía húmeda, en molino de laboratorio, con un porcentaje de sólidos entre el 65 y el $70 \%$, y una cantidad mínima de defloculante. Después de la molienda se ha completado la defloculación hasta conseguir un mínimo de viscosidad.

La barbotina, así preparada, se ha colado sobre moldes de escayola. Se han obtenido probetas cilíndricas de $1,8 \mathrm{~cm}$ de diámetro y $10 \mathrm{~cm}$ de longitud. Se han preparado 25 probetas de cada pasta, que se han separado en lotes de 5 piezas para ser cocidas a 5 temperaturas distintas, entre 1150 y $1280^{\circ} \mathrm{C}$.

Se ha realizado a cada temperatura de cocción, ensayos de contracción lineal, absorción de agua, densidad aparente y resistencia mecánica. En la pasta 2 no se realizo la cocción a $1280{ }^{\circ} \mathrm{C}$, por tenerse claro que esta temperatura era excesiva para esta composición.

Se realizan probetas finas, de varios milímetros de espesor, para observar, de forma cualitativa, la translucidez de las pastas.

Las pastas P2 y P3 presentan, a todas las temperaturas, mayor deformación que la pasta P1. Aquellas presentan a las temperaturas más elevadas, una deformación excesiva.

Se ha creído conveniente cuantificar el valor de la deformación piroplástica en aquellas pastas que podían tener problemas.

Se ha estudiado esta magnitud, en las pastas 2 y 3, a la temperatura óptima de cocción. Para ello se han confeccionado probetas prismáticas de $(25 \times 1,5 \times 1 \mathrm{~cm})$, obtenidas por colage de la barbotina sobre moldes de escayola. Las probetas se han colocado en el horno, apoyando sus extremos sobre dos soportes refractarios. Después de la cocción se ha medido la distancia entre apoyos, el espesor de la probeta y la flecha de deformación. El índice piroplástico se ha calculado mediante la fórmula:

$$
\mathrm{IP}=\frac{4 \cdot \mathrm{h}^{2} \cdot \mathrm{S}}{3 \cdot \mathrm{L}^{4}}
$$

donde IP es el índice piroplástico, y h, S y L son respectivamente, el espesor, la flecha y la distancia entre apoyos de la probeta.

\section{RESULTADOS Y DISCUSIÓN}

Tras los ensayos de cocción, estas pastas quedan muy blancas, lo que nos indica que el $\mathrm{Fe}_{2} \mathrm{O}_{3}$ presente, inferior en las tres pastas al $0,3 \%$, habrá reaccionado con el $\mathrm{P}_{2} \mathrm{O}_{5}$, para formar un compuesto volátil (5). La posible presencia de iones fluoruro, que pueden estar presentes, como trazas, en la ceniza de hue- sos (5), también ejerce acción decolorante sobre los iones férricos, por formación del ion incoloro $\left(\mathrm{FeF}_{6}\right)^{-3}$ (11).

En la tabla VI se indica la composición de las pastas cocidas P1, P2, y P3 en función de los componentes mayoritarios. Dicha composición se ha deducido a partir de análisis químico de las materias primas y de la proporción en que éstas forman parte de la pastas

TABLA VI

ANÁLISIS QUÍMICO DE LAS PASTAS COCIDAS.

\begin{tabular}{|c|c|c|c|}
\hline Componentes & P 1 & P 2 & P 3 \\
\hline $\mathrm{SiO}_{2}$ & 34,84 & 30,49 & 32,96 \\
\hline $\mathrm{Al}_{2} \mathrm{O}_{3}$ & 13,78 & 16,81 & 15,02 \\
\hline $3 \mathrm{CaO} \cdot \mathrm{P}_{2} \mathrm{O}_{5}$ & 51,38 & 52,70 & 52,02 \\
\hline
\end{tabular}

En el diagrama de fases de la figura 4 , se indica con los puntos 1,2 , y 3 , las composiciones indicadas en la tabla VI, como P1, P2 y P3.

La composición de las pastas 1 y 3 se sitúan en el campo primario de la sílice, y la pasta 2 en el campo primario del fosfato tricálcico.

Los óxidos minoritarios: $\mathrm{K}_{2} \mathrm{O}, \mathrm{Na}_{2} \mathrm{O}$ y $\mathrm{MgO}$, existentes en las pastas, que no se hacen constar, para poder representar las composiciones mencionadas en el diagrama triaxial, serán los primeros en incorporarse a la fase vítrea, y los responsables de la maduración de las pastas a temperaturas inferiores de las predichas por el diagrama de fases.

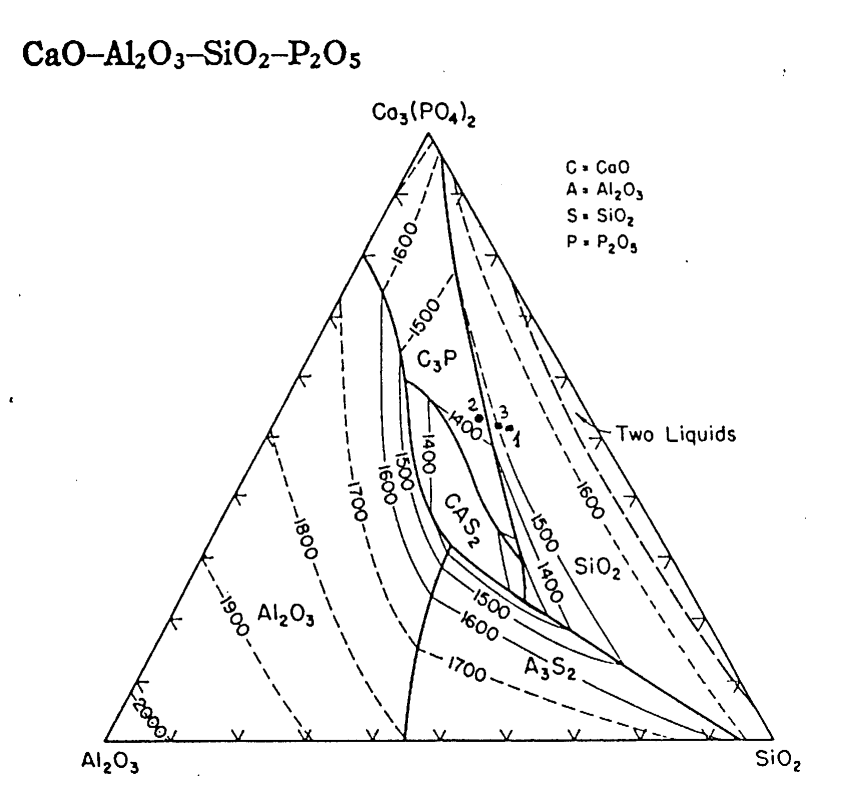

Fig 4.- Diagrama de fases del sistema: $\mathrm{Ca}_{3}\left(\mathrm{PO}_{4}\right)_{2}-\mathrm{Al}_{2} \mathrm{O}_{3}-\mathrm{SiO}_{2}$ 
En la gráfica siguiente se representa la variación de la densidad aparente en función de la temperatura para cada una de las tres pastas estudiadas.

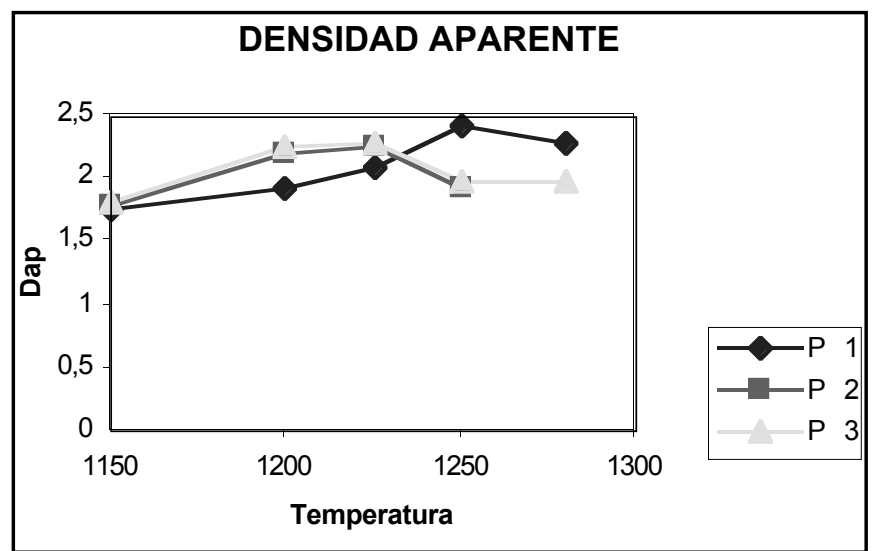

Fig. 5.- Densidad aparente de las pastas $\mathrm{P}_{1}, \mathrm{P}_{2}, \mathrm{y}_{3}$.

Los índices piroplásticos de las pastas 2 y 3 a $1225^{\circ} \mathrm{C}$ son:

$$
\begin{aligned}
& \text { Pasta } 2 \longrightarrow \mathrm{Ip}=4,10 \times 10^{-5} \\
& \text { Pasta } 3 \longrightarrow \mathrm{Ip}=2,24 \times 10^{-5}
\end{aligned}
$$

A partir de los valores obtenidos de: densidad aparente, absorción de agua, contracción lineal y resistencia mecánica, se puede afirmar lo siguiente:

Las temperaturas óptimas de cocción de las tres pastas son:

$$
\begin{aligned}
& \text { Pasta } 1-1250^{\circ} \mathrm{C} \\
& \text { Pasta } 2-1225^{\circ} \mathrm{C} \\
& \text { Pasta } 3=1225^{\circ} \mathrm{C}
\end{aligned}
$$

La resistencia mecánica de la pasta 1 es mayor que la de la pasta 3, y ésta mayor que la de la pasta 2.

La pasta 2 a $1250^{\circ} \mathrm{C}$ presenta porosidad abierta. También se observa hinchamiento.

La Pasta 1 y la pasta 3, a temperaturas superiores a las óptimas no presentan porosidad abierta.

La pasta 1 no hincha a $1280{ }^{\circ} \mathrm{C}$.

La deformación piroplástica de la pasta 3 es menor que la de la pasta 2 a la temperatura óptima de cocción de ambas pastas, $1225^{\circ} \mathrm{C}$. Es de suponer que la pasta 1 presente menor deformación piroplástica que las anteriores.

La posición de la pasta 2 en el diagrama de fases se encuentra por encima de la isoterma de $1400{ }^{\circ} \mathrm{C}$. La pasta 3 y la pasta 1 se encuentran sobre la isoterma de $1500{ }^{\circ} \mathrm{C}$ y superior, respectivamente. Esta posición en el diagrama de fases de la figura 5, unido a las cantidades de óxidos fundentes, mencionadas anteriormente, mayores en la pasta 2 , que en la 3 y mayores en ésta que en la 1; concuerdan perfectamente con la mayor deformación de la pasta 2, y con la mayor refractariedad de la pasta 1.

La resistencia mecánica de las tres pastas, a la temperatura óptima de cocción, supera en todos los casos los $800 \mathrm{Kg} / \mathrm{cm}^{2}$, y en el caso de la pasta 1 supera los $900 \mathrm{Kg} / \mathrm{cm}^{2}$. La resistencia mecánica de la porcelana de huesos es superior a la de otros tipos de porcelana.
En las gráficas siguientes se representan los datos de contracción lineal, absorción de agua y resistencia mecánica de las tres pastas.
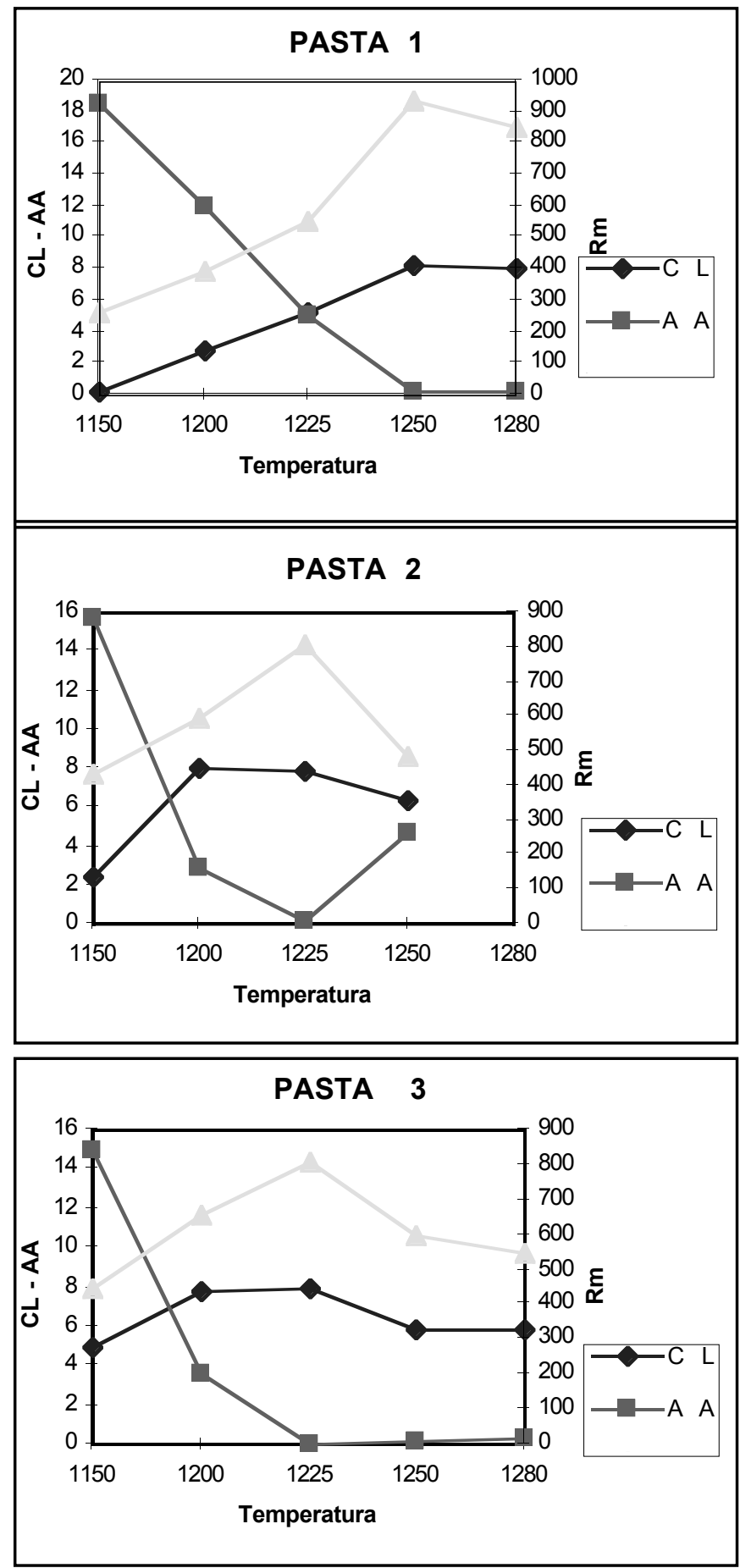

Fig. 6.- Contracción lineal (CL), absorción de agua (AA) y resistencia mecánica de las pastas $\mathrm{P}_{1}, \mathrm{P}_{2}, \mathrm{y}_{3}$. 


\section{CONCLUSIONES}

La porcelana de huesos, con respecto a otras pastas triaxiales, posee el interés didáctico de mostrar más claramente diferenciadas, las distintas etapas del proceso de sinterización.

De las tres pastas estudiadas, es la pasta 1, cuyo fundente es la pegmatita, la que presenta un mejor comportamiento.

Las pastas 2 y 3, cuyos fundentes son respectivamente la nefelina sienita y el feldespato potásico exhiben peores propiedades.

La elevada resistencia mecánica, elevada blancura y elevada translucidez, hacen que se pueda fabricar con la porcelana de huesos piezas resistentes de gran belleza.

A pesar de sus buenas propiedades, la fabricación de la porcelana de huesos no esta excesivamente generalizada, lo cual puede deberse a las siguientes causas:

La escasa plasticidad de la pasta, que conlleva baja resistencia mecánica del material crudo; todo lo cual es debido al bajo contenido de mineral arcilloso.

El riesgo de deformación del material cocido, ya que se trata de una pasta que posee un estrecho intervalo de maduración; lo cual es debido a la composición de la pasta, próxima a la composición eutéctica.

\section{BIBLIOGRAFÍA}

1.- F. H. Norton. Porcelana de huesos, Ed. Omega. Cerámica fina. España p. 272343. (1975)

2.- P. D. S. St. Pierre. "Constitution of bone china: II. Reactions in bodies". J. Amer. Soc. 38 217, (1955)

3.- H.W. Edwards. "Bone as flux in English china". Trans. English Ceram. 32, (1903 - 1904).

4.- A. S. Watts. "Bone china bodies". Trans. Amer. Ceram. Soc. 7, 204, (1905).

5.- C. García Portillo. Composición, propiedades y aplicaciones de la porcelana de huesos. Silicatos 9, 17-22, (1998).

6.- A. Dinsdale, "The Constitution of bone china", Sci. of Ceram, 3, 323, (1967).

7.- P. D. S. St. Pierre. "Constitution of bone china: I. High-Temperature Phase Equilibrium Studies in System Tricalcium Phosphate-Alumina-Silica". J. Amer. Ceram. Soc. 37, 243, (1954).

8.- H. Klause. "Bone china and its constitution". Kenam. Z. 8, 331, (1956).

9.- P. D. S. St. Pierre. “Constitution of bone china: III. High-Temperature Phase Equilibrium Studies in the System Tricalcium Phosphate-Anorthite-Silice". J. Amer. Ceram. Soc. 39, 147, (1956)

10.- D. Taylor. "A study of some of the changes in bone china and whiteware firing". Trans. Brit. Ceram. Soc. 78, 43-47, (1979).

11.- H. Salmang. Fundamentos físico-químicos de la fabricación del vidrio. Coloración por hierro, 323-331. Ed. Aguilar. Madrid (1962).

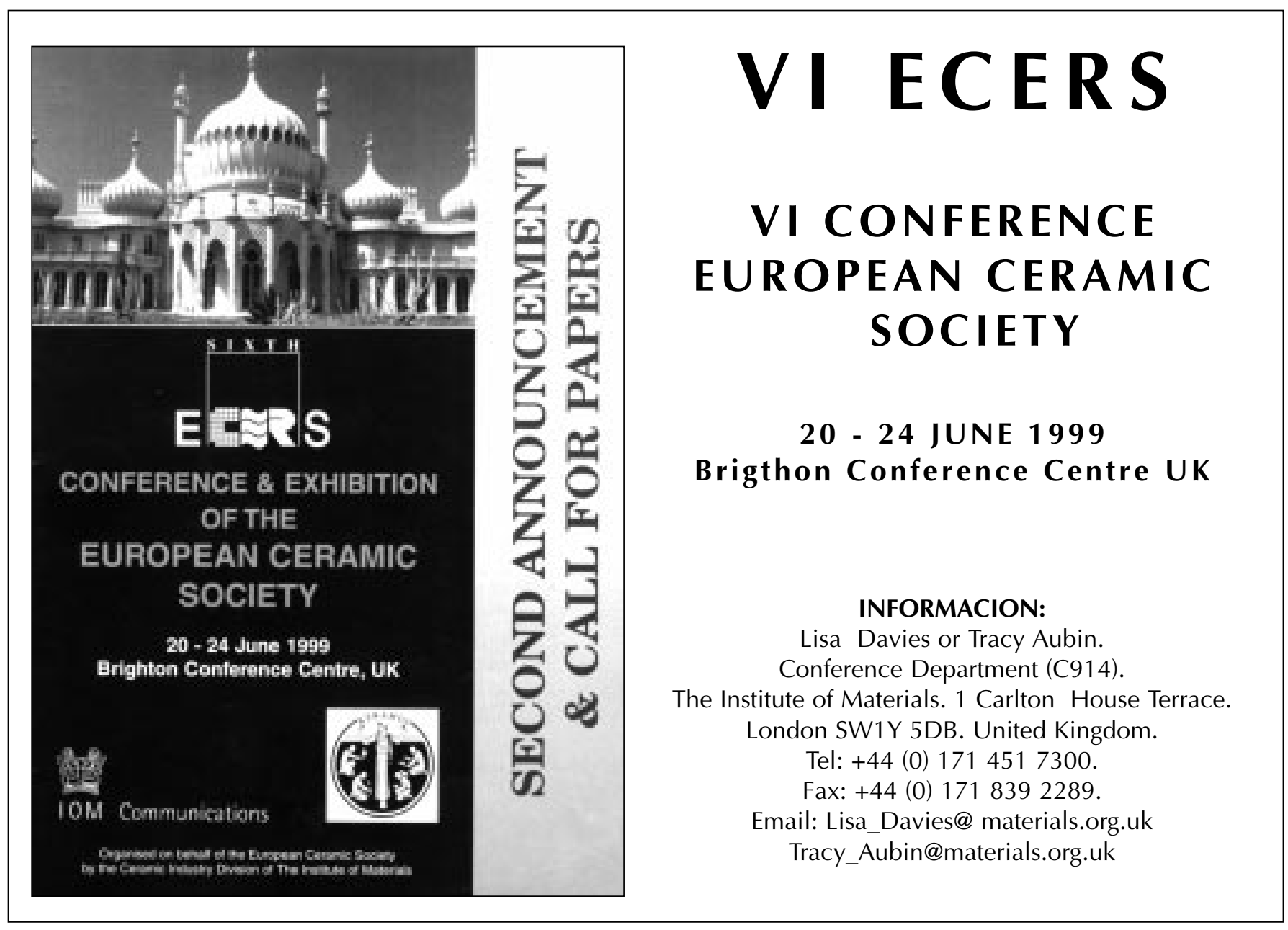

